\title{
High-Order Mesoporous (HOM) Sensors for Visual Recognition of Toxic Metal lons from Drinking Water
}

\author{
Sherif A. El-Safty ${ }^{1,2}$, M. A. Shenashen ${ }^{1}$ \\ ${ }^{1}$ National Institute for Materials Science, 1-2-1 Sengen, Tsukuba, Ibaraki, 305-0047, Japan \\ ${ }^{2}$ Graduate School for Advanced Science and Engineering, Waseda University, 3-4-1 Okubo, \\ Shinjuku-ku, Tokyo 169-8555, Japan. E-mail: sherif@aoni.waseda.jp; sherif.elsafty@nims.go.jp
}

\begin{abstract}
:
Recently, the developments of high-ordered mesoporous monoliths (HOM) open different methodological avenues for changes especially beneficial in the field of sensor applications. Growing public awareness on the potential risk to humans of toxic chemicals in the environment has generated demand for new and improved methods for toxicity assessment and removal, rational means for health risk estimation. Mesoporous materials are highly desirable materials that have a large surface area, high porosity with a highly ordered pore structure with uniform mesopores, hydrophilic character and relatively good chemical and mechanical stability was fabricated and during this study as toxic metal sensor. The result indicated high sensitivity and selectivity, also the sensor is able to serve for multiple recycle/uses.
\end{abstract}

Key words: Mesoporous, Sensor, Removal, Toxic Metal

\section{Introduction}

Manipulation of micro-sized mesoporous monoliths at the nanoscale level has enabled the development of optochemical nanosensors that exhibit high strength, as well as recognition and signaling of a broad range of chemical species [1-3]. The advanced functionality of manipulating chromophore probes into nanomaterials as sensing receptors has received attention in the design of chemical sensor arrays for responsive recognition of several species such as metal cations [4-6], and charged and neutral organic molecules $[1,2]$. As customary, its blossoming was enabled to generate novel families of fascinating reproducible sensors exhibiting a high selectivity, low time consuming, and low cost. Heavy metal ions play an important role in fundamental physiological processes in organisms ranging from bacteria to mammals. Heavy metals are metallic elements that are present in both natural and contaminated environments. In natural environments, they occur at low concentrations. It is well known that heavy metals in larger amounts are toxic to animals as well as plants; and chromium is no exception to this, though some of these metals may actually be required in trace amounts to support life. All forms of chromium have toxic properties at high levels, but chromium (VI) is very toxic. Uptake chromium with high doses has a harmful effect such as damage and irritates nose, lungs, stomach ulcers and upsets, intestines kidney and liver damage, and even death. To the best of our knowledge, no general design strategy for optochemical nanosensor arrays has been reported that incorporates simplicity in terms of fabrication time and low-cost materials but still retains sensitivity and fast-response detection of toxic metal ions [1-6]. Here, we developed the design of optical chemical nanosensors based on densely patterned a chromogenic receptor (DPC), as a selective binding site onto threedimensional (3D) nanoscale structures. Optimization of control sensing conditions was established for achieving enhanced signal response and color intensities of $\mathrm{Cr}$ (III)diphenylcarbazone complex up to subpicomolar detection limits $\left(\sim 10^{-11} \mathrm{~mol} / \mathrm{dm}^{3}\right)$ of $\mathrm{Cr}(\mathrm{VI})$ ions, for the first time, with rapid response time (in the order of seconds). The nanosensors exhibited long-term stability of signaling and recognition functionalities that in general provided extraordinary sensitivity, selectivity, reusability of $\mathrm{Cr}(\mathrm{VI})$ ions.

\section{Methodology}

We designed optochemical nanosensors for simple, high-speed detection of multiple toxic metal ions. This design strategy is an approach based on a dense pattern of immobilized hydrophobic "neutral" diphenylcarbazone 
chromophore (DPC) with intrinsic mobility via extremely robust constructed sequences onto High-order mesoporous (HOM) structures, namely, "building-blocks" without using of thiol or silane coupling agents as the modifier of the solid surfaces. For instance, the polarity of the silica surface matrices of HOM monoliths were first fine-tuned by the dispersion of cationic surfactant such as dilauryl dimethyl ammonium bromide (DDAB) (Scheme 1), leading to the formation of positively charged silica surfaces as "carriers". The ability to precisely modify the nanoscale surfaces by using a broad range of chromophores that have different molecular sizes and characteristics enables detection of toxic ions. A key feature of this building-blocks design strategy (see Fig 1) is that the surface functionality and good adsorption characteristics of the fabricated nanosensor arrays enabled the development of "pool-onsurface" sensing systems in which high flux of the metal analytes across the probe molecules was achieved without significant kinetic hindrance.

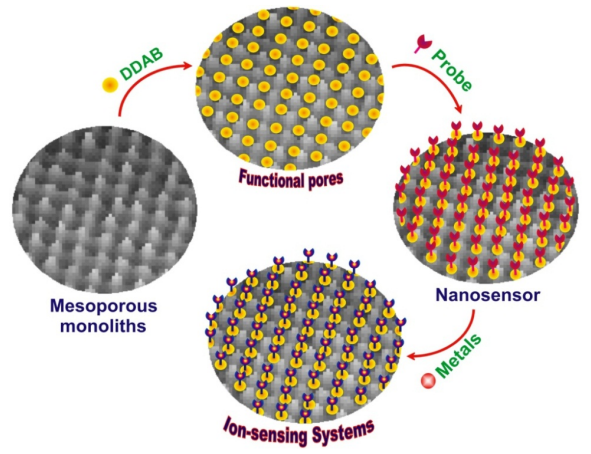

Scheme 1. Design of optical sensor based immobilizationof DPC probe into $3 D$ mesoporous silica monoliths.

\section{Results \& discussion}

A major advantage of the development nanosensor-based monoliths here is the flexibility to access the nanosacle geometries and ordered structures that have $3 \mathrm{D}$ arrays, uniform cylindrical channels, large pore sizes and volumes, high surface areas, and macroscopic particle size-like monoliths, when combined with inexpensive DPC probe molecules. Furthermore, the functional use of the optically transparent silica monoliths with cubic Fm3m (HOM-C10) structures enhanced their potential technologies as low processing costs. TEM image (Scheme1) revealed that the long-range ordered pores in large-scale domains were characteristics the optically cylindrical sensors, despite the dense construction of the building blocks.
High performance of the DPC-based nanosensor in terms of sensitivity and kinetic reaction between the metal ions and chromophore-doped HOM surfaces (timeresponse) depended on key factors such as the amount of the HOM-based sensor and the $\mathrm{pH}$ value. Changes in these control experiments can significantly affect the redistribution of the charge polarity and the electron and energy transfer within the probe molecule into the pore surfaces, permitting a high threshold level of disturbance of the sensing system. In this study, we carried out a series of experiments to systematically define and evaluate the relative importance of these factors to a HOM-Probe sensor for analyte detection (Fig. 1).

The rapid and flexible $\mathrm{Cr}(\mathrm{VI})$-DPC binding events with the formed complexes onto nanosensors led to the separation and preconcentration of $\mathrm{Cr}(\mathrm{VI})$ ions even at a trace concentration level (response-time $\leq 60 \mathrm{sec}$ ). However, the color change provided a simple procedure for sensitive, selective detection of $\mathrm{Cr}(\mathrm{VI})$ ions without the need for sophisticated instruments (Fig. 5) [30-37]. In addition, visual detection of $\mathrm{Cr}(\mathrm{VI})$ ions at a wide detection range $\left(D_{R}\right)$ of $0.07 \mathrm{ppb}-200 \mathrm{ppb}$ was clearly achieved by using 3D nanosensors.

Such a sensing design enabled sensitive recognition of metal ions up to sub-picomolar detection limits $\left(\sim 10-11 \mathrm{~mol} / \mathrm{dm}^{3}\right)$, for first time, with rapid response time within 30 seconds. Moreover, because these sensing pools exhibited long-term stability, reversibility and selectivity (see Fig 1) in detecting most pollutant cations, for example, $\mathrm{Cr}(\mathrm{VI})$ ions, they are practical and inexpensive. Key to our development of optochemical nanosensors is that although DPC chromophore moieties are commonly used for optical sensing recognition of metal ions in clean water down to a detection limit of approximately 10-6-10-8 mol/dm [4-6], our pool-like sensing systems satisfy analytical needs in terms of its sensitivity, selectivity (Fig. 1), stability, reversibility, rapid response, wide range of detectable species, and low operating cost.

A particularly important feature of the poolon surface design is the ability for responsive recognition of several cations such as $\mathrm{Cd}^{2+}$, $\mathrm{Co}^{2+}$, and $\mathrm{Pd}^{2+}$ that cannot be detected by the same optochemical nanosensors fabricated by the common grafting techniques. Moreover, our findings show evidence that pore ordering and geometry of the nanoscale structural carriers were key to improved sensing ability during the detection of analyte ions, as evidenced from the use of cage, cylindrical and worm-like pore surfaces as probe carriers. Among all possible 
interfering cation species, the $\mathrm{Pb}^{2+}$ and $\mathrm{Fe}^{3+}$ ions at high concentration showed significant change in the reflectance spectra and color intensities of complex, indicating a disturbance in the $\mathrm{Cr}(\mathrm{VI})$ ion sensing systems. To counteract such disturbance, the addition of $0.005 \mathrm{mM}$ citrate or phathalate to the specific sensing conditions can enhance the tolerable concentration of the active the $\mathrm{Pb}^{2+}$ and $\mathrm{Fe}^{3+}$ ions, respectively (Fig. 1).

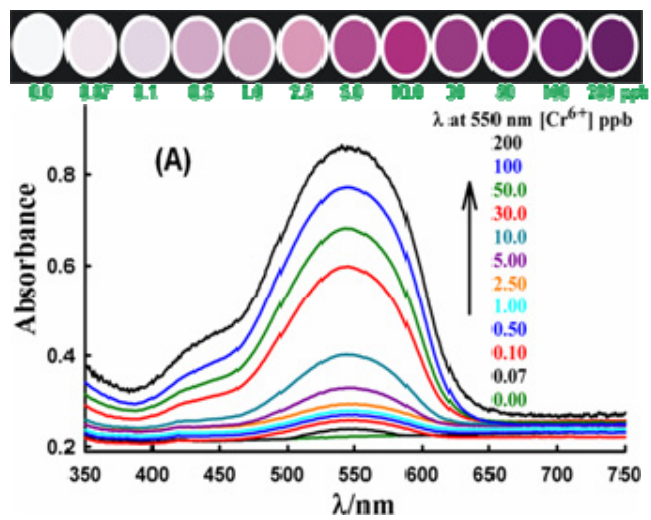

Fig. 2. A) Sensing signal responses and B- color profiles for the sensor in the presence of $\mathrm{Cr}(\mathrm{VI})$.

A major advantage of mesocaptor is their retaining functionality in terms of $\mathrm{Cr}(\mathrm{VI})$ ion recovery after multiple reuse cycles [1-6]. To investigate the efficiency of the captor design for the recovery of $\mathrm{Cr}(\mathrm{VI})$ ions after several recovery cycles, we analyzed the ICP-AES results of the $\mathrm{Cr}(\mathrm{VI})$ ions solution after completing all stages of the capture assays according to the following: 1) the removal of $\mathrm{Cr}(\mathrm{VI})$ ion from aqueous solution and 2) the release of the $\mathrm{Cr}(\mathrm{VI})$ ion from solid capture.

\section{Conclusion}

We developed the design of optical chemical nanosensors for simple, high-speed detection of toxic $\mathrm{Cr}(\mathrm{VI})$ ions. The systematic design of the optical nanosensors was based on densely patterned a chromogenic receptor (DPC), as a selective binding site onto three-dimensional (3D) nanoscale structures. The ability to precisely modify the nanoscale pore surfaces with DPC probe enables efficient sensing responses as a result of $\mathrm{Cr}(\mathrm{VI})$-DPC binding events. A key feature of this design strategy is that the pore surface-enhanced polarity by dense dispersion of cationic surfactant such as DDAB led to increased accessibility and adsorptive characteristics of the DPC probe with high intrinsic mobility systems. Such flexibility in the fabricated nanosensors led to high flux of the metal analytes across the probe molecules without significant kinetic hindrance. The synthetic nanosensor can be used for an effective recognition of $\mathrm{Cr}(\mathrm{VI})$ ions to low level of concentrations in the range of $10^{-10}-10^{-6} \mathrm{M}$ (i.e. $0.07 \mathrm{ppb}-200 \mathrm{ppb}$ ). Optimization of control sensing conditions was established for achieving enhanced signal response and color intensities of $\mathrm{Cr}(\mathrm{III})$-diphenylcarbazone complex up to sub-picomolar detection limits $\left(\sim 10^{-11} \mathrm{~mol} / \mathrm{dm}^{3}\right)$ of $\mathrm{Cr}(\mathrm{VI})$ ions, for the first time, with rapid response time (in the order of seconds). The optical chemical nanosensors are reversible and have the potential to serve effectively in on-site field analysis of environmental samples, eliminating the necessity for instrument-dependent analysis. Moreover, these new classes of the designmade hybrid nanosensors exhibited long-term stability of signaling and recognition functionalities that in general provided extraordinary sensitivity, selectivity, reusability, and fast kinetic detection and quantification of various deleterious metal ions in our environment.

Table 1. illustration of the selectivity of sensor in the presence of the competitive ions

\begin{tabular}{|c|c|c|}
\hline $\begin{array}{l}\text { Process } \\
\text { assays }\end{array}$ & {$[\mathrm{Cr}(\mathrm{VI})]$} & $\begin{array}{l}\text { Competitive lons } \\
\text { concentrations }\end{array}$ \\
\hline $\begin{array}{l}\text { Before } \\
\text { sensing }\end{array}$ & $2.0 \mathrm{ppm}$ & 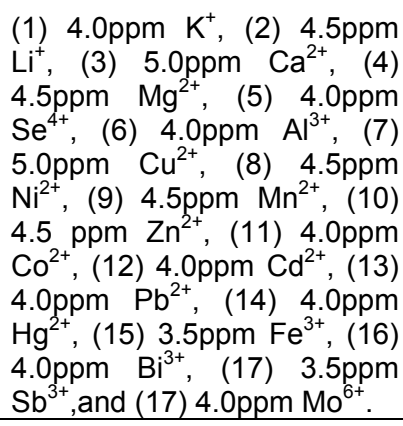 \\
\hline $\begin{array}{l}\text { After } \\
\text { Sensing }\end{array}$ & $\begin{array}{l}1.98 \\
\mathrm{ppm}\end{array}$ & 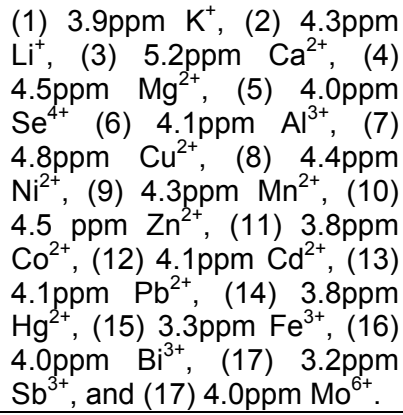 \\
\hline
\end{tabular}




\section{References}

[1] S. A. El-Safty, A. A. Ismail, A. Shahat, Optical Supermicrosensor Responses for Simple Recognition and Sensitive Removal of Cu (II) Ion Target, Talanta, 83, 1341-1351 (2011).

[2] S. A. El-Safty, Functionalized Hexagonal Mesoporous Silica Monoliths With Hydrophobic Azo- Chromophore for Enhanced Co(II) Ion Monitoring, Adsorption, 15, 227-239 (2009).

[3] S. A. El-Safty, D. Prabhakaran, Y. Kiyozumi, F. Mizukami, Nanoscale Membrane Strips for Benign Sensing of $\mathrm{Hg}$ (II) ions: A Route to Commercial Waste Treatments, Adv. Funt. Mater. 18, 1739-1750 (2008).

[4] S. A. El-Safty, A. Ismail, T. Hanaoka, H. Matsunaga, F. Mizukami, Optical Nanoscale Pool-on-Surface Design for Control Sensing Recognition of Multiple Cations, Adv. Funct. Mater. 18, 1485-1500 (2008).

[5] S. A. El-Safty, D. Prabhakaran. A. A. Ismail, H Matsunaga, F. Mizukami, Three-Dimensional Worm-like and Ordered Mesostructures and Their Applicability as Optically Ion-Sensitive Carriers, Chem. Mater. 20. 2644-2654 (2008).

[6] S. A. El-Safty, A. Ismail, H. Matsunaga, F. Mizukami,Uniformly Mesocaged Cubic Fd3m Monoliths as Modal Carriers for Optical Chemosensors, J. Phys. Chem. C, 112, 48254835 (2008).

[7] S. A. El-Safty, D. Prabhakaran. A. Ismail, H. Matsunaga, F. Mizukami, Nanosensor Design Packages: A Smart and Compact Development for Metal lons Sensing Responses, Adv. Funct. Mater. 17, 3731-3745 (2007).

[8] S. A. El-Safty, A. Ismail, H. Matsunaga, F. Mizukami, Optical nanosensor Design with Uniform Pore Geometry and Large Particle Morphology, Chem. Eur. J. 13, 9245-9255 (2007).

[9] S. A. El-Safty, T. Balaji, T. Hanaoka, H. Matsunaga, F. Mizukami, Optical Sensors-Based Nanostructured Cage Materials for Detection of Toxic Metal Ions, Angew. Chem. Int. Ed. 45, 7202- 7208 (2006). 